\title{
Solution of Two-Dimensional Free Surface Problems Based on Finite Element Program Generator
}

\author{
B. X. Wu ${ }^{1,2}, \mathrm{~S} . \mathrm{Wan}^{3}$, N. N. Chen ${ }^{2}$ \\ ${ }^{1}$ Institute of Engineering Thermophysics, Chinese Academy of Sciences, Beijing 100080, China \\ ${ }^{2}$ Beijing Fegen Software Company, Beijing 100098, China \\ ${ }^{3}$ Southeast University, Nanjing 210096, China \\ Email: wubangxian@gmail.com
}

\begin{abstract}
Computation of flow problems with moving boundaries and interfaces is one of the challenges in computational fluid mechanics. This class of problems includes flows with free surfaces, two-fluid interfaces, fluid-object and fluid-structure interactions, and moving mechanical components. Depending on the nature of the problem and the specific application requirement, different solution techniques, including interface tracking and interface capturing techniques, can be adopted. In this paper, free surface problem is solved by pseudo-concentration method. A pseudo-concentration function is defined in the entire domain to serves as a marker of the free surface. The advection equation describing the interface evolution is solved together with incompressible Navier-Stokes equation by stabilized finite element methods or fractional step methods [1].

Based on the borrowed DIY concept from hardware industry, Finite Element Program Generator (FEPG), as a platform of software development, can automatically generate Fortran program based on files in a special modeling language. Distinguished in software execution manner with general purpose commercial software, a couple of files describing the weak form of the partial differential equation for each physical field as well as a file describing the solving algorithm and procedure have to be prepared, in stead of importing parameters for the latter. It is especially superior in solving multiphysics problems and problems with complicated interfaces [2]. For solving a complicated flow problem with moving boundaries and interfaces, the flexibility of FEPG on the solver and the type of the interface condition would be advantageous to easily manipulate various interface conditions. Different solvers for any kind of free surface flow problem can be easily embedded in the program generation system by simply modifying the files mentioned above.

A dam break flow problem is exemplified to illustrate the usage and the superiority of the program generation technique in solving this class of problems, as well as to verify the effectiveness of the numerical technique. A simple interface reconstruction technique is implemented to remove the overshoots resulted from irregular values of the pseudo-concentration function during the interface evolution and to prevent the field from undesirable numerical oscillations. The result on the reduction of the water column height with time is compared with experimental data.
\end{abstract}

\section{REFERENCES}

1. Tezduyar TE. Stabilized finite element formulations for incompressible flow computations. Advances in Applied Mechanics, 1992;28:1-44

2. Wu BX, Qian HS, Wan S. Promotion of frontier science research with the aid of automatic program generation technology, In: Computational Methods in Engineering \& Science, Yao ZH, Yuan MW, Chen YQ, eds. Tsinghua University Press and Springer, 2006, 274,(CD-ROM) 